\title{
Production of nanostructures on bulk metal samples by laser ablation for fabrication of low-reflective surfaces
}

\author{
Béla Hopp • Tomi Smausz • Tamás Csizmadia • \\ Csaba Vass • Csaba Tápai · Bálint Kiss • \\ Martin Ehrhardt • Pierre Lorenz • Klaus Zimmer
}

Received: 2 August 2013 / Accepted: 3 August 2013 / Published online: 14 August 2013

(C) Springer-Verlag Berlin Heidelberg 2013

\begin{abstract}
Nanostructure formation on bulk noble metals (copper, gold and silver) by a femtosecond laser was studied aiming at the production of low-reflectivity surfaces. The target surface was irradiated with the beam of a $775 \mathrm{~nm}$ wavelength and $150 \mathrm{fs}$ pulse duration Ti:sapphire laser. The fluence was in the $16-2000 \mathrm{~mJ} / \mathrm{cm}^{2}$ range, while the average pulse number was varied between 10 and 1000 depending on the scanning speed of the sample stage. The reflectivity of the treated surfaces was measured with a visible-nearinfrared microspectrometer in the $450-800 \mathrm{~nm}$ range, while the morphology was studied with a scanning electron microscope. A strong correlation was found between the decreasing reflectivity and the nanostructure formation on the irradiated surface; however, the morphology of silver significantly differed from those of copper and gold. For the two latter metals a dense coral-like structure was found probably as a result of cluster condensation in the ablation plume followed by diffusion-limited aggregation. In the case of silver the surface was covered by nanodroplets, which formation was probably influenced by the 'spitting' caused by ambient oxygen absorption in the molten silver followed by its fast release during the resolidification.
\end{abstract}

B. Hopp $(\varangle) \cdot$ T. Csizmadia · C. Vass · C. Tápai · B. Kiss Department of Optics and Quantum Electronics, University of Szeged, 6720 Szeged, Dóm tér 9, Hungary

e-mail: bhopp@physx.u-szeged.hu

T. Smausz

MTA-SZTE Research Group on Photoacoustic Spectroscopy, University of Szeged, 6720 Szeged, Dóm tér 9, Hungary

M. Ehrhardt $\cdot$ P. Lorenz $\cdot$ K. Zimmer

Leibniz Institute of Surface Modification, Permoserstr. 15, 04318

Leipzig, Germany

\section{Introduction}

Earlier studies of laser ablation of different materials showed that femtosecond (fs) lasers are advanced tools in material processing and micromachining with high precision and negligible thermal loading to the surrounding area even in the case of metals, which have high thermal conductivity [1-3]. Therefore, the processed surfaces can have good quality on the micrometer scale. In addition, micrometerand nanometer-sized structures can be formed on the processed surface whose characteristics depend on the processed materials and the experimental conditions (fluence, ambient gas, pressure etc.). These structures can be randomlike or ordered ones [4-6] and influence the characteristics of the material's surface. For metals, the processed surfaces can show an increased absorbance in the visible and nearinfrared wavelength ranges, as compared to the untreated ones. Such a decrease of the reflectance was observed for a wide range of metals like gold, tungsten, titanium alloys, aluminum, platinum and steel and semiconductors such as germanium and silicon [7-13]. The increasing number of studies shows the high interest of researches in such patterns due to the potential application. Spectroscopic measurements revealed that the absorbance of such 'darkened metals' can reach 90-95 \% in the ultraviolet, visible and infrared wavelength ranges. The enhanced absorption of the darkened metals was attributed to nano/microscale surface structures. Since the structures are smaller than the wavelength of light, the way they reflect light is highly dependent upon their specific size and shape, which can be varied by changing the processing parameters. The femtosecond nanomachining also allowed the creation of the so-called colored metal by combining the low reflectance and light diffraction $[8,14,15]$. This was realized by combining the micro- and nanoscale features on the processed surface, i.e. 

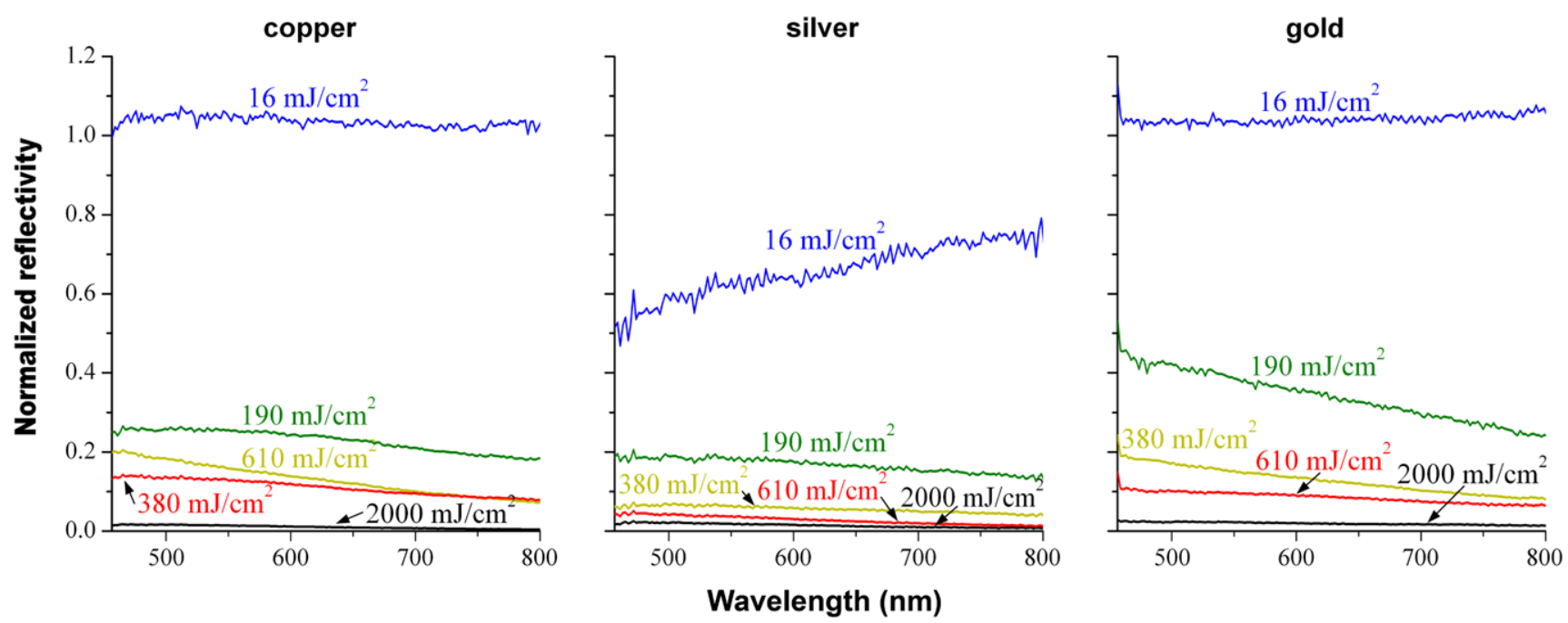

Fig. 1 Reflectivity spectra related to the untreated surface of the areas irradiated with 100 pulses at different laser fluences for the three studied metals

creating microscale lines or hole arrays covered with nanostructures arranged in regular rows, where these lines cause the diffraction of the reflected light making a piece of metal to appear of different colors in different directions.

Presumably, such metals can be used as wavelengthindependent anti-reflective surfaces darkened in their material for applications where e.g. the scattering of laser light has to be avoided, and can find application where the high efficiency and wide wavelength range radiation capturing is essential, like photovoltaic elements, light meters, laser surgery etc. The main advantage of this technique is that it does not require the application of special coatings prepared by materials which are different from the substrate material; therefore, the thermal, electronic etc. matching of these is not necessary.

Although several papers have been presented [16-18] that aim at the explanation of the absorptive properties in connection to the development process of the darkening nanostructures (disordered or laser-induced periodic structures), there are several open questions concerning both the development processes of the nanostructures and the mechanism of the reflectivity decreasing, yet. According to this in the following study we present our comparative investigations of the evolution of reflective properties in correlation with the changes of the surface morphology during femtosecond laser ablation of three different noble metals: copper, silver and gold samples.

\section{Experiments and results}

During our experiments high-purity bulk metal (copper, silver and gold) plates (from Goodfellow) were irradiated by a Ti:sapphire laser (CPA-2001, Clarc MRX: $\lambda=775 \mathrm{~nm}$,
$\mathrm{FWHM}=150 \mathrm{fs}, f=1 \mathrm{kHz}$ ). The laser beam was focused onto the sample surface in a $250 \mu \mathrm{m}$ diameter spot. The targets were placed on a scanning sample holder which enabled the irradiation of a large area by applying laterally shifted pulses. By varying the scanning speed, the pulse number reaching a given area could be varied in a 0 to 1000 range. The laser fluence was altered between 16 and $2000 \mathrm{~mJ} / \mathrm{cm}^{2}$. The experiments were carried out at atmospheric pressure at room temperature.

After the irradiation, the reflectivity change of the treated surfaces was visible by naked eye for all the applied parameter values. The reflectivity of the processed area was measured by a visible-near-infrared microspectrometer equipped with an $\mathrm{NA}=0.12$ objective, which allowed the detection of the back-scattered light in the range of 450$800 \mathrm{~nm}$. Due to the low numerical aperture, mainly the specular reflected light is measured and the scattered light is diminished. The recorded spectra normalized to the reflectivity of the untreated, non-polished metal samples can be seen in Fig. 1 for different laser fluences. The applied pulse number was 100 in this case. It can be seen that in the cases of copper and gold the measured reflectances of the illuminated areas divided by the initial values are larger than 1 at $16 \mathrm{~mJ} / \mathrm{cm}^{2}$ fluence, probably due to the removal of existing surface contaminants. In contrast with copper and gold, the normalized reflectivity of laser-treated silver decreases significantly at this fluence value. At and above $190 \mathrm{~mJ} / \mathrm{cm}^{2}$, a considerable decrease of the reflectivity is observable for all investigated metals and the tendencies of the curves are similar. It also can be seen that the reflectivity decrease is larger with increasing wavelength; therefore, the normalized spectra indicate the influence of the surface roughness on the reflectivity by multiple scattering, which effect has an increased significance with increasing wavelength [19]. 


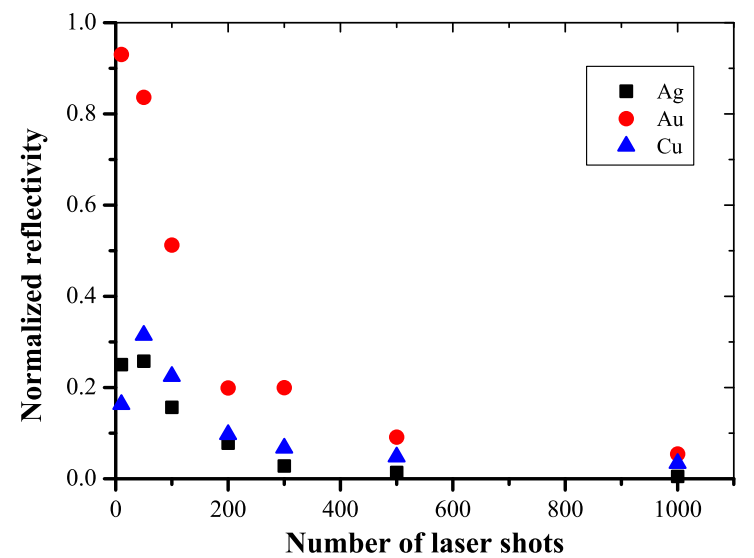

Fig. 2 Relative reflectance measured at $550 \mathrm{~nm}$ for the three metals irradiated with 100 laser pulses as a function of the applied laser fluence

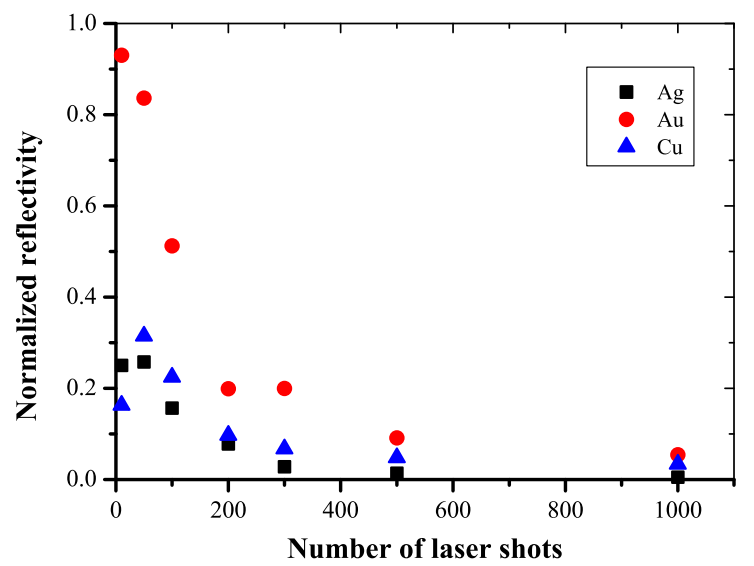

Fig. 3 Relative reflectance measured at $550 \mathrm{~nm}$ for the three metals irradiated at $190 \mathrm{~mJ} / \mathrm{cm}^{2}$ laser fluence as a function of the applied pulse number

Figure 2 shows the normalized reflectivities at $\lambda=550 \mathrm{~nm}$ in dependence on the laser fluence. The applied pulse number was 100. It was found that the normalized reflectivities decrease drastically with increasing fluence in the range of $16-500 \mathrm{~mJ} / \mathrm{cm}^{2}$. Above $800 \mathrm{~mJ} / \mathrm{cm}^{2}$ the reflectivity values diminish below $10 \%$ and their decrease slowly continues.

The dependence of the darkening of the treated spots on the applied laser pulse number was also investigated. The normalized reflectivity values at $550 \mathrm{~nm}$ wavelength as a function of the number of shots are shown in Fig. 3. The fluence was $190 \mathrm{~mJ} / \mathrm{cm}^{2}$ in this case. It can be seen clearly that a significant reflectivity decrease occurs after 10 laser pulses in the cases of copper and silver. After 100 shots the reflectivity values fell below $10 \%$ and further, but a slower decrease is observable with increasing number of pulses. In the case of the gold sample the decrease is less drastic; however, at around 1000 pulses the measured reflectivities for all the studied metals are below $5 \%$.

For the interpretation of the results we investigated the morphology of the laser-irradiated metal samples. The de- veloped surface structures were observed by a Hitachi S4700 scanning electron microscope (SEM) operating at $15 \mathrm{kV}$ accelerating voltage and with an emission current of $9 \mu \mathrm{A}$ without applying any additional conductive coating. The magnification was $50 \mathrm{k}$. Figure 4 shows the morphologies of the patterned copper, gold and silver samples after 100 pulses at different fluences. It was found that, mainly for copper and gold, morphology changes occur even at the lowest studied laser fluence, $16 \mathrm{~mJ} / \mathrm{cm}^{2}$, although this fluence value is much lower than the damage threshold values generally accepted (a few hundreds of $\mathrm{mJ} / \mathrm{cm}^{2}$ ) for these metals $[17,20,21]$. According to the reflectivity values, the observable roughness did not cause observable absorption. More significant changes in the morphology were observed when raising the fluence to the $F=190 \mathrm{~mJ} / \mathrm{cm}^{2}$ value. Above $300 \mathrm{~mJ} / \mathrm{cm}^{2}$ significant morphological differences were not observable. This tendency is in good agreement with the behavior of the measured reflectivity. It can be seen that the final structures of the illuminated $\mathrm{Cu}$ and $\mathrm{Au}$ surfaces are quite similar; however, the morphology of the treated Ag sample is very different. While on the illuminated silver surfaces a sponge-like structure formed by frozen jets and nanodroplets having basically a few hundred nanometers dimensions is visible, in the cases of the copper and gold plates a more or less similar, but less consistent, basic structure is densely covered by a mass of nano-aggregates (having approximately a few-nanometers diameter), which form a porous coral-like structure (Fig. 4).

At a fixed $190 \mathrm{~mJ} / \mathrm{cm}^{2}$ fluence we investigated the structure development process as a function of the pulse number (Fig. 5). It was found that the most significant morphological modifications of the irradiated areas took place within the first 100 shots for all the investigated metals. Above this value considerable structural changes were not observable. This is also in good agreement with the reflectivity measurements.

\section{Discussion}

Our experiments proved that femtosecond Ti:sapphire laser irradiation under the presented experimental conditions is suitable for preparation of non-reflective surface structures on copper, gold and silver bulk samples. The nature of the formed nanostructures, however, is material-dependent. It was found that the lowest value of the investigated fluence range $\left(16 \mathrm{~mJ} / \mathrm{cm}^{2}\right)$ is not enough for production of effective non-reflective surface structures, yet. In the cases of copper and gold a molten and resolidified surface structure was observed with a small amount of droplets. The measured small reflectivity increases for $\mathrm{Cu}$ and $\mathrm{Au}$ samples at $16 \mathrm{~mJ} / \mathrm{cm}^{2}$ fluence can be due to the removal of 


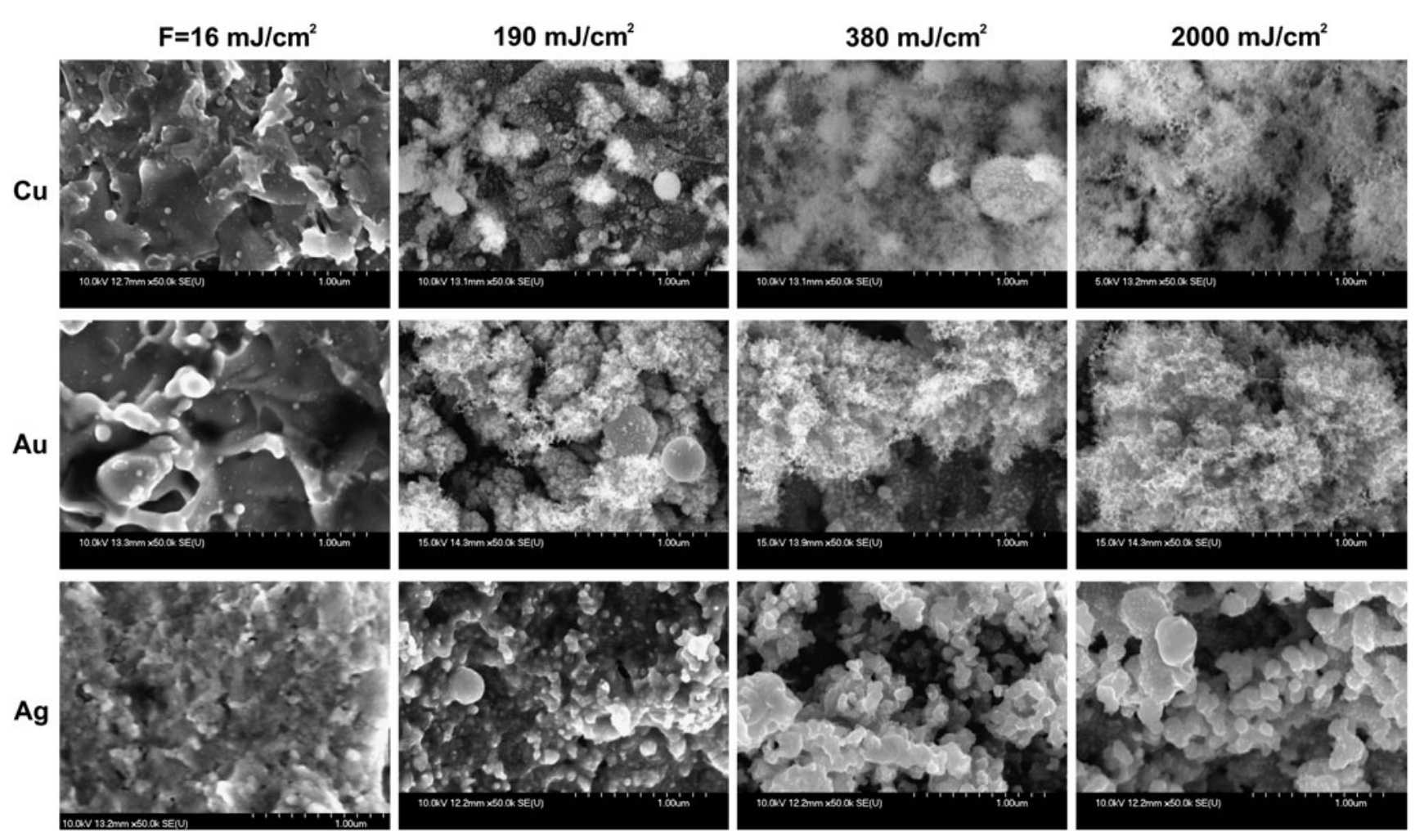

Fig. 4 Electron microscopic images of the metal surfaces irradiated by 100 pulses with different laser fluences

$\mathrm{N}=\mathbf{0}$

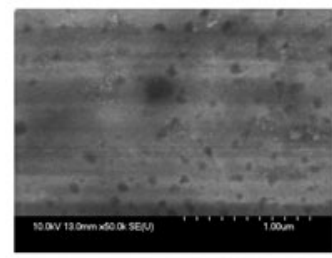

$\mathrm{Au}$

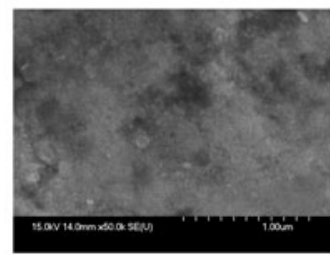

$\mathrm{Ag}$

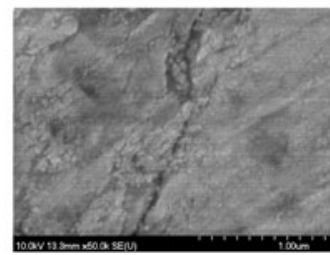

10
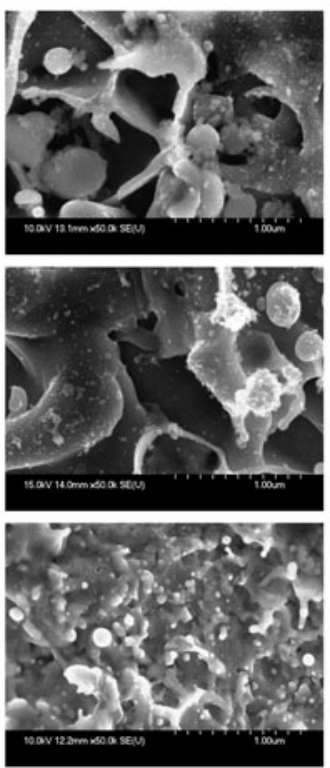

50
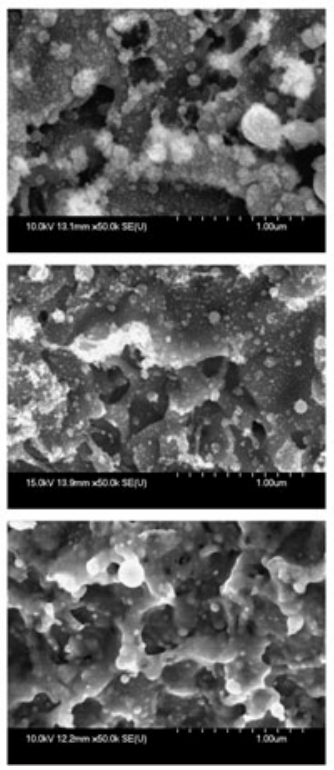

100
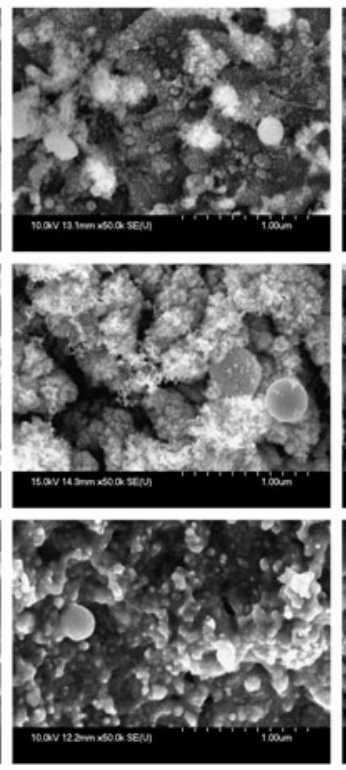

1000
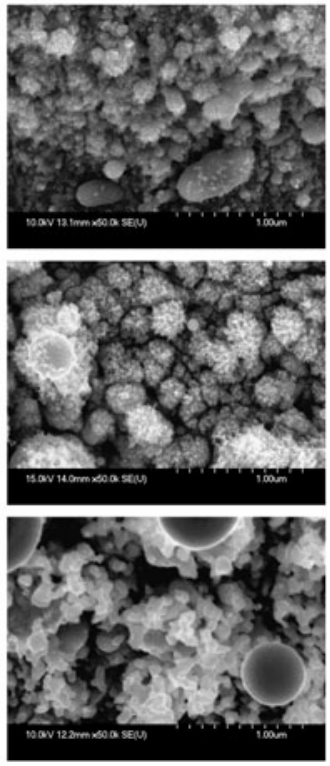

Fig. 5 Electron microscopic images of the metal surfaces irradiated with different numbers of laser pulses of $190 \mathrm{~mJ} / \mathrm{cm}^{2}$ fluence

the surface contaminants, oxides or tarnish, which can influence the reflective properties. The reflectivity of the irradiated silver surface slightly decreased in consequence of the small droplets found on the target surface probably originating from redeposition. With increasing applied laser fluence the reflectivity decreased drastically for all studied metals in consequence of the developed surface patterns.

On the basis of the recorded SEM pictures it can be seen clearly that two types of nanostructures can be responsi- 
ble for the reflectivity decrease. The lowest applied fluence $\left(16 \mathrm{~mJ} / \mathrm{cm}^{2}\right)$ is not enough to produce a considerable amount of nanoparticles, but it is enough to heat and melt the irradiated surface layer of the investigated metal samples. This means that the melting and freezing processes play an important role in the surface structure development (mainly droplets and frozen jets are observable in the SEM pictures), while the influence of the back-scattered and deposited nanoparticles is negligible in this case. At $190 \mathrm{~mJ} / \mathrm{cm}^{2} \mathrm{flu}-$ ence the importances of the two above-mentioned effects are similar (droplets, jets and nanoclusters are visible), while above this value the role of the deposited nanoclusters becomes dominant.

As an approximation, the melting of the surface requires absorbed fluences of around 7,5 and $4.5 \mathrm{~mJ} / \mathrm{cm}^{2}$ for copper, gold and silver, respectively, while these values are about 10 times higher for the evaporation condition. Taking into account that the surface absorbance is more significant even for the untreated samples at high femtosecond intensities than at the low-regime values [17], the $16 \mathrm{~mJ} / \mathrm{cm}^{2}$ and the $190 \mathrm{~mJ} / \mathrm{cm}^{2}$ incident fluences can assure the conditions for melting and boiling, respectively. This is also confirmed by a detailed study of fs copper ablation, where a low-fluence ablation regime with a threshold of $18 \mathrm{~mJ} / \mathrm{cm}^{2}$ was found [22].

The supposed optical mechanisms causing the reduced reflectance of the darkened metal surface are summarized and discussed by Vorobyev and Guo [17], where the evolution of surface morphology and the reflectivity properties of a fs laser treated copper surface was studied. It is noticeable that the basic surface morphology of the copper was similar to that observed in our experiments; however, the foam-like deposit was completely missing from their structure. This difference can be attributed to the different experimental arrangements used. In the setup of Ref. [17] a fixed copper target was irradiated, while we applied continuous target scanning. It is plausible that the copper foam originated from the redeposited material ablated from a nearby area. At high fluences (around $190 \mathrm{~mJ} / \mathrm{cm}^{2}$ and above in our case) in the ablation plume the interaction with the high-pressure ambient gas leads to particle condensation and cluster formation near the irradiated spot in the early expansion regime when the ablation plume is dense enough [23]. For this reason the femtosecond ablation can be used as a nanoparticle generating method [24-26]. Because of collisions within the plume itself and with the background gas molecules, a certain percentage of ejected particles (atoms) is back reflected and moves back towards the target surface [27]. Monte Carlo simulations of Morozov proved that up to $20 \%$ of atoms could be back reflected depending on the background pressure [28]. Égerházi et al. also demonstrated that this backward scattering of the ablated fragments has practical application possibility for example in thin-layer deposition (inverse PLD) [29]. Depending on the quantity of the clus- ters and nanoparticles deposited on the surface, a diffusionlimited aggregation can start forming a cluster-assembled coral-like structure, as shown by Rode et al. when ablating glassy carbon with a high repetition rate femtosecond laser [23].

Our other observation that has to be discussed is the absence of the coral-like structure from the treated silver samples. Since it was valid in the whole fluence range the differences in optical and thermal properties can hardly be a possible explanation. It may be related to the fact that in experiments aiming at metal nanoparticle generation by laser ablation in ambient atmosphere significant differences were observed in the size distribution of the related metals [2426]: while the sizes of copper and gold nanoparticles were well below $10 \mathrm{~nm}$, which corresponds to the particle range forming the aggregates in our experiments, the size of silver nanoparticles is $\sim 100 \mathrm{~nm}$, which corresponds to the size of the particles covering our laser-treated silver samples. One possible explanation of this behavior could be the high oxygen solubility at high temperature in molten silver. It is known that above the melting point silver can absorb an oxygen quantity corresponding to 20 times its volume at $1 \mathrm{~atm}$ pressure. During cooling the oxygen desorbs quickly, resulting in the phenomenon called silver 'spitting'. This could result in the formation and ejection of silver nanodroplets in the ablation plume. A certain part of these back reflects and redeposits onto the target surface forming the observed sponge-like nanostructure.

\section{Summary}

During our experiments bulk copper, silver and gold samples were irradiated by a femtosecond Ti:sapphire laser at different fluences and with selected numbers of pulses to produce low-reflective surface nanostructures. The reflectivity of the laser-treated areas was studied and it was found that its normalized value diminishes drastically with increasing fluence in the range of $16-500 \mathrm{~mJ} / \mathrm{cm}^{2}$, while above $800 \mathrm{~mJ} / \mathrm{cm}^{2}$ the decrease slowly continues for all the investigated metals. Further investigations demonstrated that a significant reflectivity decrease occurs after 10 laser pulses and at around 1000 shots the normalized reflectivity values diminish below $5 \%$. The scanning electron microscopic investigations proved that while on the irradiated silver surfaces spongelike structures formed by frozen jets and nanodroplets having basically a few hundred nanometers dimensions are visible, in the cases of the copper and gold samples a more or less similar, but less consistent, basic structure is densely covered by a mass of nanofibres (having approximately a few-nanometers diameter), which form foam-like structures. In the development of the sponge-like pattern the ejection and falling back of molten silver droplets having several 
tens nanometers diameter and jets play an important role, while the forming of the observed foam-like structure can be due to the back scattering and aggregation of $\mathrm{Cu}$ and $\mathrm{Au}$ nanoparticles with few-nanometers dimensions.

Acknowledgements This work was partially supported by the European Union and co-financed by the European Social Fund through projects 'Impulse lasers for use in materials science and biophotonics' (grant no. TÁMOP-4.2.2.A-11/1/KONV-2012-0060) and 'Biological and environmental responses initiated by new functional materials' (grant no. TÁMOP-4.2.2.A-11/1/KONV-2012-0047) and the DAAD (no. 56266271).

\section{References}

1. S. Preuss, A. Demchuk, M. Stuke, Appl. Phys. A 61, 33 (1995)

2. C. Momma, S. Nolte, B.N. Chichkov, F.V. Alvensleben, A. Tünnermann, Appl. Surf. Sci. 109-110, 15 (1997)

3. J. Békési, J.-H. Klein-Wiele, P. Simon, Appl. Phys. A 76, 355 (2003)

4. N. Singh, D.R. Alexander, J. Schiffern, D. Doerr, J. Laser Appl. 18(3), 242 (2006)

5. B.K. Nayak, M.C. Gupta, K.W. Kolasinski, Nanotechnology 18, $195302(2007)$

6. J. Wang, C. Guo, Appl. Phys. Lett. 87, 251914 (2005)

7. A.Y. Vorobyev, C. Guo, Adv. Mech. Eng. 2010, 452749 (2010)

8. A.Y. Vorobyev, C. Guo, Opt. Photonics News 19(12), 30 (2008)

9. A.Y. Vorobyev, C. Guo, Phys. Rev. B 72(19), 195422 (2005)

10. Y. Vorobyev, C. Guo, J. Appl. Phys. 104, 053516 (2008)
11. T.-F. Yao, P.-H. Wu, T.-M. Wu, C.-W. Cheng, S.-Y. Yang, Microelectron. Eng. 88, 2908 (2011)

12. R. Torres, V. Vervisch, M. Halbwax, T. Sarnet, P. Delaporte, M. Sentis, J. Ferreira, D. Barakel, S. Bastide, F. Torregrosa, H. Etienne, L. Roux, J. Optoelectron. Adv. Mater. 12(3), 621 (2010)

13. A.Y. Vorobyev, C. Guo, Opt. Express 19(5), A1031 (2011)

14. J. Yao, C. Zhang, H. Liu, Q. Dai, L. Wu, S. Lan, A.V. Gopal, V.A. Trofimov, T.M. Lysak, Appl. Surf. Sci. 258, 7625 (2012)

15. M.S. Ahsan, F. Ahmed, Y.G. Kim, M.S. Lee, M.B.G. Jun, Appl. Surf. Sci. 257(17), 7771 (2011)

16. A.Y. Vorobyev, C. Guo, Appl. Phys. A 86, 321 (2007)

17. A.Y. Vorobyev, C. Guo, J. Appl. Phys. 110, 043102 (2011)

18. A.Y. Vorobyev, C. Guo, Appl. Phys. A 86, 235 (2007)

19. K.-E. Peiponent, T. Tsuboi, Opt. Laser Technol. 22(2), 127 (1990)

20. E.G. Gamaly, Phys. Rep. 508, 91 (2011)

21. J. Byskov-Nielsen, J.-M. Savolainen, M.S. Christensen, P. Balling, Appl. Phys. A 101, 97 (2010)

22. M. Hashida, A. Semerok, O. Govert, G. Petite, Y. Izawa, J.F. Wagner, Appl. Surf. Sci. 197-198, 862 (2002)

23. A.V. Rode, E.G. Gamaly, B. Luther-Davies, Appl. Phys. A 70, 135 (2000)

24. S. Noël, J. Hermann, T. Itina, Appl. Surf. Sci. 253(15), 6310 (2007)

25. P. Spiga, J. Hermann, T. Itina, D. Grojo, D. Neamtu, D. Pailharey, W. Marine, AIP Conf. Proc. 799, 197 (2005)

26. S. Barcikowski, A. Hahn, A.V. Kabashin, B.N. Chichkov, Appl. Phys. A 87, 47 (2007)

27. M. Biscan, S. Milosevic, Spectrochim. Acta B 68, 34 (2012)

28. A.A. Morozov, Appl. Phys. A 79, 997 (2004)

29. L. Égerházi, Zs. Geretovszky, T. Szörényi, Appl. Surf. Sci. 247, $182(2005)$ 